\title{
Smoking is a negative predictor of arteriovenous malformation posttreatment obliteration: analysis of vascular risk factors in 774 patients
}

\author{
Richard Dalyai, M.D., ${ }^{1}$ Robert M. Starke, M.D., ${ }^{2}$ Nohra Chalouhi, M.D., ${ }^{1}$ \\ Thana Theofanis, M.D., ${ }^{1}$ Christopher Busack, ${ }^{1}$ Pascal Jabbour, M.D., ${ }^{1}$ \\ L. Fernando Gonzalez, M.D. ${ }^{3}$ Robert Rosenwasser, M.D., ${ }^{1}$ \\ and STaVropoula TJoumakaris, M.D. ${ }^{1}$ \\ ${ }^{1}$ Department of Neurosurgery, Thomas Jefferson University and Jefferson Hospital for Neuroscience, \\ Philadelphia, Pennsylvania; ${ }^{2}$ Department of Neurosurgery, University of Virginia Health System, \\ Charlottesville, Virginia; and ${ }^{3}$ Department of Neurosurgery, Duke University, Durham, North Carolina
}

\begin{abstract}
Object. Cigarette smoking has been well established as a risk factor in vascular pathology, such as cerebral aneurysms. However, tobacco's implications for patients with cerebral arteriovenous malformations (AVMs) are controversial. The object of this study was to identify predictors of AVM obliteration and risk factors for complications.

Methods. The authors conducted a retrospective analysis of a prospectively maintained database for all patients with AVMs treated using surgical excision, staged endovascular embolization (with $N$-butyl-cyanoacrylate or Onyx), stereotactic radiosurgery (Gamma Knife or Linear Accelerator), or a combination thereof between 1994 and 2010. Medical risk factors, such as smoking, abuse of alcohol or intravenous recreational drugs, hypercholesterolemia, diabetes mellitus, hypertension, and coronary artery disease, were documented. A multivariate logistic regression analysis was conducted to detect predictors of periprocedural complications, obliteration, and posttreatment hemorrhage.

Results. Of 774 patients treated at a single tertiary care cerebrovascular center, 35\% initially presented with symptomatic hemorrhage and $57.6 \%$ achieved complete obliteration according to digital subtraction angiography (DSA) or MRI. In a multivariate analysis a negative smoking history (OR 1.9, $\mathrm{p}=0.006$ ) was a strong independent predictor of AVM obliteration. Of the patients with obliterated AVMs, $31.9 \%$ were smokers, whereas $45 \%$ were not $(\mathrm{p}=0.05)$. Multivariate analysis of obliteration, after controlling for AVM size and location (eloquent vs noneloquent tissue), revealed that nonsmokers were more likely (0.082) to have obliterated AVMs through radiosurgery. Smoking was not predictive of treatment complications or posttreatment hemorrhage. Abuse of alcohol or intravenous recreational drugs, hypercholesterolemia, diabetes mellitus, and coronary artery disease had no discernible effect on AVM obliteration, periprocedural complications, or posttreatment hemorrhage.

Conclusions. Cerebral AVM patients with a history of smoking are significantly less likely than those without a smoking history to have complete AVM obliteration on follow-up DSA or MRI. Therefore, patients with AVMs should be strongly advised to quit smoking.

(http://thejns.org/doi/abs/10.3171/2014.5.FOCUS14121)
\end{abstract}

KEY WoRdS - arteriovenous malformation • smoking • hemorrhage

A LTHOUGH intracranial arteriovenous malformations (AVMs) cause a relatively small proportion of strokes, they are a significant etiology of hemorrhagic stroke (approximately 49\%) in younger patients. ${ }^{10}$ Modifiable risk factors for ischemic stroke have been identified, such as hypertension and diabetes mellitus. ${ }^{3,7,17}$ Identification of these medical risk factors has been instrumental in delineating areas for treatment and behavioral modifications toward the prevention of ischemic stroke. Unfortunately, however, the medical risk factors for poor

\footnotetext{
Abbreviations used in this paper: AVM = arteriovenous malformation; DSA = digital subtraction angiography; $\mathrm{SM}=$ SpetzlerMartin; SRS = stereotactic radiosurgery.
}

treatment outcomes and complications in cerebral AVMs are relatively unknown. Arteriovenous malformations can be treated with open microsurgical removal, endovascular embolization, stereotactic radiosurgery (SRS), or a combination thereof. We investigated the relative rates of AVM obliteration, treatment outcome, and hemorrhagic complications with regard to significant medical comorbidities. From these data, we attempted to define modifiable risk factors that could become potential targets for medical therapy or patient behavioral counseling.

\section{Methods}

Institutional review board approval was obtained prior 
R. Dalyai et al.

to data collection. We searched a prospectively maintained database at our tertiary care cerebrovascular center for all patients with AVMs that had been treated using surgical excision, endovascular embolization (with $\mathrm{N}$-butylcyanoacrylate or Onyx), stereotactic radiosurgery (Gamma Knife or Linear Accelerator), or a combination thereof between 1994 and 2010. We reviewed history and physical examinations, progress notes, discharge summaries, operative reports, and clinical follow-up notes for each patient. We determined a patient's smoking history based on his or her documentation of active smoking at the time of presentation or treatment; that is, at the clinical encounter the patient admitted to smoking, which we documented on their history. We defined nonsmokers as patients who had never smoked or had a minimal and remote smoking history. All other patients were excluded from the analysis. Medical risk factors were identified, such as a history of cigarette smoking, social history including abuse of alcohol or intravenous recreational drugs, coronary artery disease, and active treatment for hypercholesterolemia, diabetes mellitus, or hypertension. Besides these modifiable risk factors, patient age, lesion location (eloquent or noneloquent tissue), lesion size, and hemorrhagic presentation were recorded. Patients with coronary artery disease were defined as those who had a significant history of cardiac surgery with cardiac stents or bypass grafts. Patients with hyperlipidemia were defined as those currently being treated with cholesterol-lowering medications. Similarly, patients with hypertension or diabetes were defined as those receiving blood pressure- or serum glucose-lowering medications, respectively. Patient charts as well as MRI, CT, and cerebral digital subtraction angiography (DSA) studies were systematically analyzed to identify AVM obliteration and patient complications (both procedural and postoperative).

Data are presented as the mean and range for continuous variables and as frequency for categorical variables. Analysis was performed using the unpaired t-test, chisquare test, and Fisher's exact test. The means of more than two groups were assessed using ANOVA with post hoc analysis, with Bonferroni correction as appropriate. Univariate analysis was used to test covariates predictive of the following dependent variables: new or worsening neurological deficit following treatment, hemorrhage following treatment, and AVM obliteration. Interaction and confounding were assessed through stratification and relevant expansion covariates. Factors predictive in univariate analysis $(\mathrm{p}<0.15)$ were entered into a multivariate logistic regression analysis. A p value $\leq 0.05$ was considered statistically significant. Statistical analysis was performed with Stata 10.0 (StataCorp).

\section{Results}

Seven hundred seventy-four patients with AVMs treated using embolization, SRS, and/or surgery were included in this analysis (Tables 1 and 2). There were 385 female patients $(49.7 \%)$. The median age was 42.5 years. Two hundred seventy-one patients $(35 \%)$ presented with hemorrhage. Ninety patients $(11.6 \%)$ were treated for medically intractable seizures. Seventy-two patients $(27 \%)$ had a significant smoking history (only 263 patients had
TABLE 1: Summary of characteristics in 774 patients with AVMs*

\begin{tabular}{|c|c|c|}
\hline Characteristic & No. & $\%$ \\
\hline \multicolumn{3}{|l|}{ sex } \\
\hline M & 389 & 50.3 \\
\hline $\mathrm{F}$ & 385 & 49.7 \\
\hline median age in yrs & 42.5 & \\
\hline \multicolumn{3}{|l|}{ age group } \\
\hline $0-21$ yrs & 76 & 9.9 \\
\hline $22-40$ yrs & 245 & 31.4 \\
\hline $41-50$ yrs & 185 & 23.8 \\
\hline $51-60$ yrs & 156 & 20.2 \\
\hline$>60 \mathrm{yrs}$ & 112 & 14.6 \\
\hline \multicolumn{3}{|c|}{ hemorrhagic presentation } \\
\hline no & 503 & 65.0 \\
\hline yes & 271 & 35.0 \\
\hline \multicolumn{3}{|l|}{ intractable seizures } \\
\hline no & 684 & 88.4 \\
\hline yes & 90 & 11.6 \\
\hline \multicolumn{3}{|l|}{ smoking history $\dagger$} \\
\hline no & 191 & 72.6 \\
\hline yes & 72 & 27.4 \\
\hline \multicolumn{3}{|c|}{ significant cardiac surgery } \\
\hline no & 717 & 92.6 \\
\hline yes & 57 & 7.4 \\
\hline \multicolumn{3}{|l|}{ hyperlipidemia } \\
\hline no & 706 & 91.2 \\
\hline yes & 68 & 8.8 \\
\hline \multicolumn{3}{|l|}{ hypertension } \\
\hline no & 652 & 84.2 \\
\hline yes & 122 & 15.8 \\
\hline \multicolumn{3}{|l|}{ lesion size } \\
\hline small $(<3 \mathrm{~cm})$ & 436 & 56.3 \\
\hline medium $(3-6 \mathrm{~cm})$ & 303 & 39.1 \\
\hline large $(>6 \mathrm{~cm})$ & 35 & 4.5 \\
\hline \multicolumn{3}{|l|}{ lesion location } \\
\hline noneloquent tissue & 226 & 29.2 \\
\hline eloquent tissue & 548 & 70.8 \\
\hline \multicolumn{3}{|l|}{ venous drainage } \\
\hline superficial & 373 & 48.2 \\
\hline deep & 401 & 51.8 \\
\hline \multicolumn{3}{|l|}{ SM grade } \\
\hline I & 90 & 11.7 \\
\hline ॥ & 216 & 27.8 \\
\hline III & 282 & 36.4 \\
\hline IV & 158 & 20.4 \\
\hline V & 28 & 3.6 \\
\hline
\end{tabular}

* $\mathrm{SM}=$ Spetzler-Martin.

† Only 263 patients had data regarding smoking history. 


\section{Smoking is a negative predictor of AVM posttreatment obliteration}

TABLE 2: Treatment type for AVMs in 774 patients

\begin{tabular}{lc}
\hline \multicolumn{1}{c}{ Treatment } & No. of Patients \\
\hline op excision only & 152 \\
SRS only & 178 \\
embolization + op excision & 103 \\
embolization + SRS & 244 \\
embolization + SRS + op excision & 11 \\
embolization only & 86 \\
\hline
\end{tabular}

data regarding smoking history). One hundred ninety-one (73\%) patients had clearly documented a negative smoking history. Fifty-seven patients (7.4\%) had significant cardiac surgery. Sixty-eight patients $(8.8 \%)$ were actively being treated for hyperlipidemia. One hundred twenty-two patients (15.8\%) were being treated for hypertension. Four hundred thirty-six patients (56\%) had small AVMs, 303 (39\%) had medium AVMs, and 35 (5\%) had large AVMs. Five hundred forty-eight AVMs (71\%) were located in eloquent areas. Four hundred one AVMs (51.8\%) had deep venous drainage. Spetzler-Martin grades are listed in Table 1.

Three hundred sixteen $(57.6 \%)$ of 549 patients with available follow-up imaging had complete obliteration according to DSA or MRI. In multivariate analysis, predictors of total AVM obliteration were a history of open surgery, no history of smoking, and a lower SM grade (Table 3 ). While $45 \%$ of nonsmokers (86 patients) had obliterated AVMs, only $31.9 \%$ of those with a smoking history (23 patients) had obliterated AVMs $(\mathrm{p}=0.05)$. A negative smoking history was associated with a 1.9 times greater likelihood of complete AVM obliteration. When obliteration was analyzed by treatment modality, controlling for AVM size and location, nonsmokers were more likely to have obliterated AVMs with SRS treatment ( $\mathrm{p}=0.08$; Table 4). Among the 30 patients with significant symptomatic posttreatment hemorrhage, multivariate analysis showed embolizations and presentation of hemorrhage as positive predictors (Table 5). Smoking was not predictive of AVM complications or posttreatment hemorrhage. Abuse of alcohol or intravenous recreational drugs, hypercholesterolemia, diabetes mellitus, and coronary artery disease had no statistically significant effect on AVM obliteration, periprocedural complications, or posttreatment hemorrhage.

\section{Discussion}

Smoking is a well-established risk factor in vascular pathology. With regard to cerebral aneurysms, smoking is a major risk factor for rupture and growth of residual aneurysms after treatment. ${ }^{9}$ In one analysis, it was determined that approximately $21 \%$ of subarachnoid hemorrhage cases would not occur if the risk factor of smoking were eliminated from the population. ${ }^{9}$ Similarly, with regard to ischemic stroke, cigarette smokers have a 2-4 times higher risk than nonsmokers. ${ }^{13}$ However, since smoking impairs endogenous tissue plasminogen activator release and causes circulating fibrinogen levels to rise, ${ }^{1,11}$ this fibrin-rich thrombus in smokers has been shown to be $4-6$ times more likely to respond to fibrinolytic therapy, resulting in recanalization. ${ }^{6}$ While significant literature exists on cigarette smoking's effects on aneurysmal subarachnoid hemorrhage and ischemic stroke, there is a relative lack of understanding of how smoking may affect patients with cerebral AVMs.

\section{Cigarette Smoking Pathophysiology}

Cigarette smoke from burning tobacco is composed of around 5000 chemicals. Its components create effects from nondilute gases such as carbon monoxide, carbon dioxide, and nitrogen dioxide, as well as from soluble particles such as nicotine, phenol, polyaromatic hydrocarbons, and nitrosamines contained in the particulate phase, which can enter the bloodstream. ${ }^{4}$ Several toxins associated with cigarette smoke, such as nicotine, carbon monoxide, reactive oxygen species, and acrolein, have significant inflammatory and immunomodulatory potential. Passive smoking, or exposure to environmental tobacco smoke, has also been shown to increase the risk of cardiovascular disease including ischemic stroke.

\section{Role of SRS in AVMs}

Some researchers have hypothesized that risk factors for systemic vascular disease, such as cigarette smok-

TABLE 3: Multivariate analysis of predictors for complete AVM obliteration*

\begin{tabular}{lcccc}
\hline \multicolumn{1}{c}{ Factor } & OR & SE & $p$ Value & $95 \% \mathrm{Cl}$ \\
\hline eloquent tissue location & 0.808 & 0.72 & 0.812 & $0.140-4.650$ \\
deep venous drainage & 1.460 & 0.41 & 0.681 & $0.240-8.880$ \\
lesion size & 0.833 & 0.74 & 0.839 & $0.144-4.797$ \\
lower SM grade & 0.697 & 0.07 & 0.000 & $0.571-0.852$ \\
embolization & 0.894 & 0.07 & 0.181 & $0.759-1.053$ \\
\hline open surgical removal & 8.852 & 2.15 & 0.000 & $5.489-14.277$ \\
SRS & 0.740 & 0.12 & 0.069 & $0.535-1.023$ \\
nonsmoker & 1.936 & 0.46 & 0.006 & $1.211-3.086$ \\
hemorrhagic presentation & 1.127 & 0.25 & 0.592 & $0.720-1.752$ \\
cardiac surgery & 1.489 & 0.42 & 0.168 & $0.845-2.622$ \\
hypertension & 1.32 & 0.28 & 0.179 & $0.877-2.001$ \\
\hline
\end{tabular}

* Shading represents significant factors. SE = standard error. 
TABLE 4: Multivariate analysis of predictors for complete obliteration of AVMs treated with SRS

\begin{tabular}{lcccc}
\hline \multicolumn{1}{c}{ Factor } & OR & SE & $p$ Value & $95 \% \mathrm{Cl}$ \\
\hline nonsmoker & 1.70 & 0.520 & 0.082 & $0.93-3.09$ \\
lesion size & 0.60 & 0.133 & 0.021 & $0.39-0.92$ \\
eloquent tissue location & 0.43 & 0.149 & 0.015 & $0.22-0.85$ \\
embolization & 0.95 & 0.303 & 0.861 & $0.50-1.77$ \\
age >65 yrs & 0.99 & 0.009 & 0.167 & $0.97-1.00$ \\
hemorrhagic presentation & 0.68 & 0.192 & 0.178 & $0.40-1.19$ \\
\hline
\end{tabular}

ing, can lead to atherosclerosis and may facilitate AVM obliteration, especially when treated with SRS. ${ }^{2}$ Research has shown that SRS initially affects AVMs with damage to endothelial cells and the intimal layer thickening by proliferation of myofibroblasts..$^{12,14,15}$ Later changes of cellular degeneration, hyaline transformation, fragmentation of the elastic lamina, and mineralization in vessel walls result in progressive stenosis and luminal closure, which eventually lead to obliteration of the AVM nidus. ${ }^{5}$

\section{AVM Hemorrhage Risk Factors}

Many factors have been reported to be associated with the risk of AVM hemorrhage, such as age, sex, clinical presentation at treatment, and history of hemorrhage. ${ }^{18}$ Authors of one study have also focused on and discussed morphological features of AVMs as predictors of hemorrhage. ${ }^{18}$ However, few studies have related modifiable risk factors to treatment success and complications. This is the largest study thus far to analyze the correlation between medical and/or vascular risk factors and cerebral AVM obliteration, complications, and hemorrhage.

\section{Present Study}

In this study we assessed the outcomes and complications of the treatment of cerebral AVMs and their correlation with medical risk factors. Overall the morbidity and mortality rates in our patient series are comparable with those in previous AVM series. ${ }^{16}$ The most significant conclusion of our study is that smoking is a negative predictor of treatment success for cerebral AVMs with an OR of 1.9. In comparison, an OR of 1.9 is similar in scale to the effect of smoking on ischemic stroke or aneurysmal subarachnoid hemorrhage. Our finding of the deleterious effect of smoking contradicts the prior theoretical assumption that smoking may attenuate the vascular effects of SRS. ${ }^{2}$ Similarly, Quigg et al. ${ }^{8}$ found that hypertension, patient sex, and tobacco use did not convey additional risk of radiation toxicity from SRS to patients with AVMs.

Looking at our subpopulation of patients treated with SRS, we found that smoking tended to be a negative predictor of AVM obliteration. We have two potential hypotheses to explain this trend. First, our study includes a large population of patients who were treated with some form of endovascular embolization (445 patients), and the vascular immune/inflammatory response associated with smoking may have deterred the endothelialization effect induced by embolization. Second, we defined nonsmokers as those who had never smoked. Patients who had smoked regularly but in years past and those without clear documentation of active smoking were removed from our analysis.

\section{Study Limitations}

Our study is limited by its retrospective design and recall bias. Identification of risk factors relied on retrospective documentation. Moreover, given the limitations of the retrospective review, we were unable to conduct an intention-to-treat analysis between the different modalities. Because posttreatment CT was not routinely performed, clinically silent hemorrhages and infarcts may have been underestimated. Since this study was performed at a single neurovascular center, it is limited by the patient demographics and population characteristics of this geographic region.

\section{Conclusions}

This study shows that patients with a history of smok-

TABLE 5: Multivariate analysis of predictors for posttreatment hemorrhage*

\begin{tabular}{lcccc}
\hline \multicolumn{1}{c}{ Factor } & OR & SE & $p$ Value & $95 \% \mathrm{Cl}$ \\
\hline smoking & 0.594 & 0.240 & 0.197 & $0.269-1.310$ \\
lesion size & 1.477 & 0.886 & 0.516 & $0.455-4.786$ \\
eloquent tissue location & 5.096 & 6.074 & 0.172 & $0.492-52.690$ \\
SM grade & 0.975 & 0.437 & 0.956 & $0.404-2.350$ \\
embolization & 1.480 & 0.146 & 0.011 & $1.220-1.790$ \\
SRS & 1.072 & 0.234 & 0.749 & $0.698-1.647$ \\
asymptomatic presentation & 1.698 & 0.757 & 0.235 & $0.708-4.067$ \\
nonhemorrhagic presentation & 0.362 & 0.150 & 0.015 & $0.160-0.819$ \\
\hline
\end{tabular}

* Shading represents significant factors. 


\section{Smoking is a negative predictor of AVM posttreatment obliteration}

ing and treated with any modalities for cerebral AVMs are almost 2 times less likely than their nonsmoking counterparts to have complete AVM obliteration. Therefore, patients with cerebral AVMs should be systematically questioned and strongly advised to quit smoking.

\section{Disclosure}

The authors report no conflict of interest concerning the materials or methods used in this study or the findings specified in this paper. Dr. Jabbour is a consultant for ev3, Codman, and Mizuho; Dr. Tjoumakaris is a consultant for Stryker; Dr. Gonzalez is a consultant for ev3; and Dr. Rosenwasser is a consultant for Boston Scientific.

Author contributions to the study and manuscript preparation include the following. Conception and design: Tjoumakaris, Dalyai. Acquisition of data: Tjoumakaris, Dalyai, Theofanis. Analysis and interpretation of data: Tjoumakaris, Dalyai. Drafting the article: Tjoumakaris, Chalouhi, Busack. Critically revising the article: Tjoumakaris, Dalyai, Jabbour, Gonzalez, Rosenwasser. Reviewed submitted version of manuscript: Tjoumakaris, Dalyai. Approved the final version of the manuscript on behalf of all authors: Tjoumakaris. Statistical analysis: Starke, Busack. Study supervision: Tjoumakaris.

\section{References}

1. Barua RS, Sy F, Srikanth S, Huang G, Javed U, Buhari C, et al: Acute cigarette smoke exposure reduces clot lysis-association between altered fibrin architecture and the response to t-PA. Thromb Res 126:426-430, 2010

2. Bhatnagar A, Flickinger JC, Kondziolka D, Niranjan A, Lunsford LD: An analysis of the effects of smoking and other cardiovascular risk factors on obliteration rates after arteriovenous malformation radiosurgery. Int J Radiat Oncol Biol Phys 51:969-973, 2001

3. Burchfiel CM, Curb JD, Rodriguez BL, Abbott RD, Chiu D, Yano K: Glucose intolerance and 22-year stroke incidence. The Honolulu Heart Program. Stroke 25:951-957, 1994

4. Chalouhi N, Ali MS, Starke RM, Jabbour PM, Tjoumakaris SI, Gonzalez LF, et al: Cigarette smoke and inflammation: role in cerebral aneurysm formation and rupture. Mediators Inflamm 2012:271582, 2012

5. Chang SD, Shuster DL, Steinberg GK, Levy RP, Frankel K: Stereotactic radiosurgery of arteriovenous malformations: pathologic changes in resected tissue. Clin Neuropathol 16:111-116, 1997

6. Kufner A, Nolte CH, Galinovic I, Brunecker P, Kufner GM, Endres M, et al: Smoking-thrombolysis paradox: recanalization and reperfusion rates after intravenous tissue plasminogen activator in smokers with ischemic stroke. Stroke 44:407-413, 2013

7. MacMahon S, Rodgers A: Blood pressure, antihypertensive treatment and stroke risk. J Hypertens Suppl 12:S5-S14, 1994
8. Quigg M, Yen CP, Chatman M, Quigg AH, McNeill IT, Przybylowski CJ, et al: Risks of history of diabetes mellitus, hypertension, and other factors related to radiation-induced changes following Gamma Knife surgery for cerebral arteriovenous malformations. Clinical article. J Neurosurg 117 Suppl:144-149, 2012 (Erratum in J Neurosurg 118:1154, 2013)

9. Ruigrok YM, Buskens E, Rinkel GJ: Attributable risk of common and rare determinants of subarachnoid hemorrhage. Stroke 32:1173-1175, 2001

10. Ruíz-Sandoval JL, Cantú C, Barinagarrementeria F: Intracerebral hemorrhage in young people: analysis of risk factors, location, causes, and prognosis. Stroke 30:537-541, 1999

11. Sambola A, Osende J, Hathcock J, Degen M, Nemerson Y, Fuster V, et al: Role of risk factors in the modulation of tissue factor activity and blood thrombogenicity. Circulation 107: 973-977, 2003

12. Schneider BF, Eberhard DA, Steiner LE: Histopathology of arteriovenous malformations after gamma knife radiosurgery. J Neurosurg 87:352-357, 1997

13. Shah T, Swerdlow D: Detecting, predicting and modifying cardiovascular risk: new and developing strategies. Expert Rev Cardiovasc Ther 8:1519-1521, 2010

14. Steinberg GK, Chang SD, Levy RP, Marks MP, Frankel K, Marcellus M: Surgical resection of large incompletely treated intracranial arteriovenous malformations following stereotactic radiosurgery. J Neurosurg 84:920-928, 1996

15. Szeifert GT, Timperley WR, Forster DM, Kemeny AA: Histopathological changes in cerebral arteriovenous malformations following Gamma Knife radiosurgery. Prog Neurol Surg 20: 212-219, 2007

16. van Beijnum J, van der Worp HB, Buis DR, Al-Shahi Salman R, Kappelle LJ, Rinkel GJ, et al: Treatment of brain arteriovenous malformations: a systematic review and meta-analysis. JAMA 306:2011-2019, 2011

17. Wolf PA, Benjamin EJ, Belanger AJ, Kannel WB, Levy D, D'Agostino RB: Secular trends in the prevalence of atrial fibrillation: the Framingham Study. Am Heart J 131:790-795, 1996

18. Yamada S, Takagi Y, Nozaki K, Kikuta K, Hashimoto N: Risk factors for subsequent hemorrhage in patients with cerebral arteriovenous malformations. J Neurosurg 107:965-972, 2007

Manuscript submitted April 14, 2014.

Accepted May 14, 2014.

Please include this information when citing this paper: DOI: 10.3171/2014.5.FOCUS14121.

Address correspondence to: Stavropoula I. Tjoumakaris, M.D., Department of Neurological Surgery, Division of Neurovascular Surgery and Endovascular Neurosurgery, Thomas Jefferson University Hospital, 909 Walnut St., 2nd Floor, Philadelphia, PA 19107. email: stavropoula.tjoumakaris@jefferson.edu. 